\title{
Síndrome de Realimentación: Revisión de tema
}

\section{Re-feeding Syndrome: A review}

Pág. 85,94

Recibido: $10-03-2020$

Aceptado: 12-09-2020

Dr. Yoel Merenstein Hoffman 1

Dra. Mariana Villalobos Jímenez 2

Dra. Camila Castro Durán 3

Dr. Ricardo Camacho Morale 4

Dr. Fabian Rodriguez Palma 5

1,2,3. Médico General, Trabajador independiente, San José Costa Rica

4,5. Médico General, Caja costarricense del seguro social, San José Costa Rica

\section{FINANCIAMIENTO}

La presente investigación no ha recibido ayudas específicas provenientes de agencias del sector público, sector comercial o entidades sin ánimo de lucro.

\section{RESUMEN}

El síndrome de realimentación (SR) se encuentra constituido por una serie de alteraciones metabólicas, producidas por el reinicio de la alimentación posterior a un periodo prolongado de ayuno. Durante el periodo de ayuno, el cuerpo entra en un estado de metabolismo catabólico; el cual posteriormente se convierte en un estado anabólico con la reintroducción de la alimentación. Dicho proceso explica las distintas alteraciones hidroelectrolíticas.

La prevención constituye el pilar de tratamiento en este padecimiento, el NICE ha elaborado una serie de criterios para agrupar a las personas según el riesgo. Entre los pacientes con mayor riesgo se encuentran aquellos que presentan alguna patología oncológica, enfermedad crónica, trastornos alimenticios, entre otros. EI SR requiere de un manejo precoz basado en un adecuado diagnóstico y la correcta administración tanto calórica como electrolítica según cada caso.

\section{ABSTRACT}

The refeeding syndrome (SR) is constituted by a series of metabolic alterations, produced by the reintroduction of feeding after a prolonged period of fasting. During the fasting period the body enters in a state of catabolic metabolism; which later becomes an anabolic state with the reintroduction of food. This process explains the different hydroelectrolytic alterations. 
Prevention is the mainstay of treatment in this condition, the NICE has developed a series of criteria to group people according to risk. Among the patients with the highest risk are those who present some oncological pathology, chronic disease, eating disorders, among others. SR requires early management based on an adequate diagnosis and the adequate caloric and electrolytic administration according to each case.

\section{PALABRAS CLAVES}

Síndrome de realimentación, ayuno, hipofosfatemia, tratamiento, prevención.

\section{KEY WORDS}

Refeeding syndrome, fasting, hypophosphatemia, treatment, prevention.

\section{INTRODUCCIÓN}

El síndrome de realimentación (SR) es definido como la agrupación de desórdenes metabólicos producidos por el reinicio del soporte nutricional, ya sea por vía oral, parenteral o enteral, en individuos considerados severamente desnutridos o los cuales han tenido periodo de ayuno durante un tiempo establecido $(1,2)$.

Dicho síndrome fue descrito inicialmente en prisioneros de la II Guerra Mundial en quienes se sometía ayuno casi total durante periodos largos de tiempo, posterior a su liberación, se reiniciaba la alimentación, en donde se observaba alteraciones a nivel cardiaco y neurológico, causando inclusive muerte súbita, posteriormente se documentó el mismo fenómeno con el inicio de la nutrición parenteral en pacientes desnutridos, donde se administró soporte nutricional de manera agresiva, se realizó estudios entre los cuales destacan el experimento de Minnesota, donde se sometía a restricción alimentaria a voluntarios por 6 meses y realimentación posterior observándose mismo cuadro descrito en dicha guerra, pero de forma más leve (3).

Dentro de las alteraciones metabólicas más importantes destacan la hipofosfatemia severa, hipokalemia, hipomagnesemia, déficit de tiamina, retención de agua y sodio, entre otros. Los pacientes que se encuentran en riesgo para desarrollar el SR son los adultos mayores con diferentes comorbilidades, anorexia nerviosa, pacientes con alteración en la absorción de nutrientes (intestino corto, fibrosis quística) $(2,4)$.

La prevalencia e incidencia de SR ha sido variable, alcanzando rangos de prevalencia de entre $0,43 \%$ a $34 \%$, por lo que no se conoce con exactitud, dentro de la razones principales se encuentra el subdiagnóstico de SR, además de los diferentes criterios utilizados para diagnosticar el síndrome, muchos estudios definen como tal el SR con presencia se hipofostatemia, por lo que las cifras son altas, sin embargo, no siempre se encontrará este hallazgo en los pacientes con SR, lo cual se dificulta aún más su estimación epidemiológica (5).

Se han establecido pautas para la prevención adecuada de SR dirigidas por NICE (National Institute for Health and Care Excellence), quienes recomiendan una evaluación nutricional completa anterior a iniciar el soporte nutricional, además de una reposición estructurada y adecuada según los requerimientos individuales de cada paciente, así como su correcto control posterior a la reposición (6). 


\section{FISIOPATOLOGÍA}

En condiciones fisiológicas, los hidratos de carbono -reservas de glucógeno hepático y muscular-, fungen como la fuente primaria de energía para los tejidos. Cuando el cuerpo se enfrenta a un ayuno, se generan cambios en el metabolismo y en la regulación hormonal -disminución en la secreción de distintas hormonas- , con el fin de preservar las funciones vitales (3).

Durante el ayuno el cuerpo entra en un estado catabólico, en donde los niveles plasmáticos de insulina disminuyen y aumentan los niveles de glucagón, dando como resultado una conversión acelerada del glucógeno en glucosa; mediante este proceso se utilizará el glucógeno hasta agotar su reserva (7).

Como consecuencia de la deprivación de las reservas de glucógeno se inicia con la proteólisis para posteriormente realizarse gluconeogénesis (8). Sumado a los procesos anteriormente descritos, los adipocitos liberan ácidos grasos y glicerol, para ayudar a mantener las distintas vías metabólicas. Si el ayuno se prolonga, llega un momento en el que los ácidos grasos libres y los cuerpos cetónicos pasan a ser la fuente principal de energía.

Como consecuencia del estado catabólico, se produce una pérdida de masa magra en el organismo $(5,8-10)$.

Durante el periodo de ayuno, se produce una disminución en la secreción de distintas hormonas, se da un descenso del tono simpático que conlleva a una disminución cercanaal $25 \%$ del metabolismo basal $(10,11)$.
Además de la pérdida de peso y de masa muscular, se da una reducción en el volumen celular de algunos órganos $(3,8)$. A nivel hidroelectrolítico se presentan alteraciones por depleción de potasio, magnesio y fósforo; aunque las concentraciones séricas pueden estar falsamente normales durante la realimentación, al ser iones predominantemente intracelulares $(3,7,10)$.

Al iniciar la realimentación sucede lo contrario al periodo de ayuno, se da un estado anabólico. Esto conlleva a un aumento en la secreción de insulina generando una entrada de iones al espacio intracelular. Sumado al aumento en la secreción de insulina, se produce una activación de la síntesis de proteínas, lípidos y glucógeno.

Esta síntesis requiere de múltiples cofactores como el fósforo, magnesio y tiamina; por lo que se exacerba la deficiencia de estos electrolitos a nivel sérico $(3,5,8,12)$. La introducción rápida de carbohidratos puede llevar a la disminución en la excreción de sodio y agua, generando un aumento del volumen extracelular; por lo que se generará una sobrecarga de volumen que se puede manifestar como edema pulmonar, edema periférico o descompensación cardiaca $(8,11,13)$.

\section{MANIFESTACIONES CLÍNICAS}

Los síntomas del SR son variables, inespecíficos, en la mayoría de casos impredecibles y su tiempo de aparición puede ir desde minutos hasta horas. La aparición de la sintomatología se debe a los trastornos hidroelectrolíticos que ocurren, los cuales generan un desbalance que conlleva a cambios en el potencial de

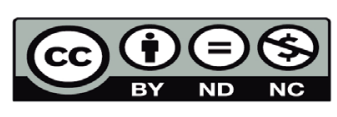


membrana en diferentes células del cuerpo tales como neuronas, células cardiacas y de músculo esquelético (11).

La variabilidad clínica se relaciona directamente con los electrolitos que se encuentran afectados y la severidad de su trastorno; de modo que, en los casos en que los cambios son mínimos, usualmente los pacientes pueden cursar asintomáticos, mientras que en pacientes con cambios más marcados la clínica puede ser incluso mortal (14). Cabe destacar que en estos pacientes la fórmula y la composición de las soluciones que se utilicen para su realimentación es un factor que influye directamente en la clínica, dado que puede empeorar en muchos casos la sintomatología y definir un importante número de las manifestaciones que se reflejan debido a los mecanismos mediante los cuales funcionan (5).

En la mayoría de los casos, los pacientes pueden presentarse con un cuadro de sintomatología inespecífica y leve tal como náuseas, vómitos, letargo; sin embargo, no es infrecuente que debuten con síntomas más severos tales como insuficiencia respiratoria, falla cardiaca, hipotensión, arritmias, delirio, coma y hasta muerte. El deterioro clínico puede ocurrir rápidamente si la causa no se establece y se demora en tomar las medidas necesarias para su corrección (14).

Las manifestaciones se pueden dar a nivel de diferentes sistemas y con gravedades variables. En el cuadro 1, se cita las principales manifestaciones clínicas a nivel de cada sistema según el trastorno hidroelectrolítico asociado.

Cuadro I. Manifestaciones clínicas del Síndrome de Realimentación $(12,14)$

\begin{tabular}{|c|c|}
\hline Sistema & Manifestación Clínica \\
\hline \multirow[t]{18}{*}{ Cardiovascular } & Hipofosfatemia $\left(\mathrm{PO}_{4}{ }^{2-}\right)$ : \\
\hline & Arritmias, Hipotensión, \\
\hline & Shock Cardiogénico. \\
\hline & Hipocalemia $\left(\mathrm{K}^{+}\right)$: \\
\hline & Hipotensión, Arritmias, \\
\hline & Bradi o Taquicardia, \\
\hline & Paro cardiaco. \\
\hline & Hipomagnesemia \\
\hline & $\left(\mathrm{Mg}^{2+}\right)$ : Arritmias, \\
\hline & Alteraciones en la \\
\hline & repolarización. \\
\hline & Hiponatremia $\left(\mathrm{Na}^{+}\right)$: \\
\hline & Arritmia, Falla \\
\hline & cardiaca; Insuficiencia \\
\hline & cardiaca. \\
\hline & En todos los casos \\
\hline & puede presentarse \\
\hline & muerte súbita. \\
\hline \multirow[t]{10}{*}{ Gastrointestinal } & Hipocalemia $\quad\left(\mathrm{K}^{+}\right)$: \\
\hline & Náuseas, \\
\hline & Vómitos, Anorexia, Íleo \\
\hline & paralítico, \\
\hline & Constipación. \\
\hline & Hipomagnesemia \\
\hline & $\left(\mathrm{Mg}^{2+}\right)$ : \\
\hline & abdominal, Diarrea o \\
\hline & Constipación, Vómitos, \\
\hline & Anorexia. \\
\hline
\end{tabular}




\begin{tabular}{|c|c|}
\hline Musculoesquelético & Hipofosfatemia $\left(\mathrm{PO}_{4}{ }^{2-}\right)$ : \\
\hline & Debilidad, Mialgias, \\
\hline & $\begin{array}{l}\text { Rabdomiolisis, Falla } \\
\text { diafragmática. }\end{array}$ \\
\hline & Hipocalemia \\
\hline & Debilidad, Fatiga, \\
\hline & Espasmos musculares. \\
\hline & Hipomagnesemia \\
\hline & $\left(\mathrm{Mg}^{2+}\right): \quad$ Debilidad, \\
\hline & Fatiga, Calambres. \\
\hline & Hiponatremia $\quad\left(\mathrm{Na}^{+}\right)$: \\
\hline & Calambres, Fatiga, \\
\hline & Retención de fluidos y \\
\hline & edema. \\
\hline Endocrino & Hipofosfatemia $\left(\mathrm{PO}_{4}{ }^{2-}\right)$ : \\
\hline & Hiperglicemia, \\
\hline & Resistencia a la \\
\hline & Insulina, Osteomalacia. \\
\hline Respiratorio & Hipocalemia \\
\hline & Hipoventilación, \\
\hline & Distress \\
\hline & $\begin{array}{l}\text { respiratorio, } \quad \text { Falla } \\
\text { ventilatoria. }\end{array}$ \\
\hline & Hipomagnesemia \\
\hline & $\left(\mathrm{Mg}^{2+}\right)$ : Hipoventilación, \\
\hline & Distress respiratorio, \\
\hline & Falla ventilatoria. \\
\hline & Hiponatremia $\quad\left(\mathrm{Na}^{+}\right)$: \\
\hline & ventilatoria, \\
\hline & Edema pulmonar. \\
\hline
\end{tabular}

\begin{tabular}{|ll|}
\hline Neurológico & Hipofosfatemia $\left(\mathrm{PO}_{4}{ }^{2}\right):$ \\
& Delirio, Coma, \\
& Convulsiones, Tetania \\
& Hipomagnesemia \\
& $\left(\mathrm{Mg}^{2+}\right)$ : Ataxia, Vértigo, \\
& Parestesias, \\
& Alucinaciones, \\
& Depresión, \\
& Convulsiones. \\
& Hiponatremia $\left(\mathrm{Na}^{+}\right):$ \\
& Deficiencia de tiamina: \\
& Síndrome de Wernicke- \\
& Korsakoff, Psicosis de \\
& Korsakoff. \\
\hline Hetabólico & Hipocalemia $\quad\left(\mathrm{K}^{+}\right):$ \\
& Alcalosis metabólica \\
\hline Hematológico & Hipofosfatemia $\left(\mathrm{PO}_{4}{ }^{2-}\right):$ \\
& Hemólisis, \\
& Trombocitopenia, \\
& Disfunción leucocitaria \\
& Anemia. \\
\hline & Hipofosfatemia $\left(\mathrm{PO}_{4}{ }^{2}\right):$ \\
Necrosis tubular aguda, & Acidosis metabólica. \\
\hline
\end{tabular}

Dentro de las principales alteraciones hidroelectrolíticas se encuentra la hipofosfatemia ( $<0.80 \mathrm{mmol} / \mathrm{L}$ ), la cual depende exclusivamente del grado de desnutrición que presente el paciente y no de la energía que se utilice para la realimentación (8). Dicha alteración puede ocurrir sumamente rápido, incluso tras un lapso 
de 48 horas de malnutrición severa y se asocia con disminución en la supervivencia de estos pacientes. La reposición del fósforo se puede realizar vía oral si la sintomatología del paciente lo permite y de no ser posible se debe realizar vía intravenosa (8).

El magnesio es un ion sobre todo intracelular, cofactor fundamental para varias enzimas e involucrado en el adecuado funcionamiento de la membrana celular. Bajos niveles de magnesio $(<0.70 \mathrm{mmol} / \mathrm{L})$ pueden evocar diferente sintomatología, dentro de lo que más se debe tener presente son las arritmias cardiacas potencialmente letales. Su reposición puede ser realizada vía oral, sin embargo, en la actualidad, lo más recomendado es la reposición intravenosa, con monitoreo cercano de los niveles plasmáticos para garantizar una adecuada corrección y evitar la aparición de hipermagnesemia (8).

En el caso del potasio, dicho catión es fundamental para la adecuada función de la membrana celular y para el correcto balance ácido base. La hipocalemia ( $<3.5 \mathrm{mmol} / \mathrm{L})$ es una de las alteraciones electrolíticas más comunes del SR y a su vez se encuentra asociada con múltiples manifestaciones clínicas. En estos casos la reposición se recomienda que se realice por vía intravenosa, a una velocidad de infusión no mayor a $20 \mathrm{mmol} / \mathrm{h}$. Es importante también tener presente y realizar el manejo de la hipomagnesemia en los casos de hipocalemia refractaria (8).

Cabe resaltar que independientemente del electrolito que se encuentre alterado, siempre es importante realizar un adecuado diagnóstico diferencial, dado que puede haber múltiples causas distintas al SR; siempre tomando en cuenta lo fundamental de la identificación temprana de la causa y su manejo (8).

\section{Prevención}

El primer paso en la prevención y el manejo del SR, se basa en la identificación de aquellos pacientes que se encuentran en mayor riesgo de padecerlo. En el cuadro II se detallan los criterios establecidos por el Instituto Nacional de Excelencia en Salud y Cuidado (NICE por sus siglas en inglés), en el año 2006 para reconocer a los pacientes con un alto riesgo de desarrollar un SR y que, por tanto, deberían mantenerse bajo una monitorización más estricta, una vez que se inicia con el proceso de nutrir al paciente $(6,10)$.

Cuadro II. Criterios para identificar pacientes con alto riesgo de síndrome de realimentación según la NICE (6)

\section{Presencia de 1 o más de los siguientes} criterios (criterios mayores):

- $I M C<16 \mathrm{~kg} / \mathrm{m}^{2}$.

- Pérdida de peso no intencional $>15 \%$ en los últimos 3-6 meses.

- Poca o ninguna alimentación por $>10$ días.

- Niveles bajos de potasio, fosfato o magnesio previo a la realimentación.

\section{2 o más de los siguientes criterios}

(criterios menores):

- $I M C<18,5$ kg/m².

- Pérdida de peso no intencional $>10 \%$ en los últimos 3-6 meses.

- Poca o ninguna alimentación por >5 días.

- Historia de abuso de alcohol, drogas (incluyendo insulina), quimioterapia, antiácidos o diuréticos. 
Actualmente, los expertos mantienen como base los mismos criterios creados por el NICE para identificar a los pacientes propensos a presentar un SR; sin embargo, subdividieron los grupos de riesgo en bajo, alto y muy alto riesgo. De modo que, un paciente de bajo riesgo es aquel que cumple únicamente con 1 criterio menor y un paciente de alto riesgo aquel que cumple con 2 criterios menores o 1 criterio mayor. Por su parte, para definir al paciente de muy alto riesgo se adicionaron las siguientes pautas: IMC $<14$ $\mathrm{kg} / \mathrm{m} 2$, poca o nula alimentación por $>15$ días, pérdida de peso $>20 \%$ en un lapso 3-6 meses $(15,16)$.

Es importante tener presente que, debido a las características clínicas asociadas a ciertas patologías, los pacientes oncológicos, con enfermedades crónicas, de edad avanzada, con cirugías recientes, trastornos alimenticios, alcoholismo crónico, síndromes malabsortivos o malnutrición crónica; se encuentran más propensos a cumplir con los criterios mencionados anteriormente y por tanto, a sufrir de SR. Resulta imprescindible entonces, que estos grupos sean sometidos a una valoración médica y nutricional adecuadas antes de iniciar con el proceso de realimentación. Dicha evaluación debe incluir una adecuada historia clínica, examen físico y una valoración completa del estado de hidratación del paciente y de su balance hidroelectrolítico, con el objetivo de categorizar correctamente al paciente $(16,17)$.

Una vez que se ha identificado a algún paciente con riesgo de padecer SR, se recomienda llevar a cabo una serie de medidas preventivas, con el fin de disminuir el riesgo de desarrollarlo. A continuación, se describirán los principales pasos a seguir para lograr dicho objetivo:

La distribución de la dieta debe componerse de
$40-60 \%$ carbohidratos, $30-40 \%$ grasas y $15-20 \%$ proteínas en los 3 grupos de riesgo a lo largo de todo el proceso. Dicho aporte calórico puede ser administrado por cualquier vía de alimentación (15).

En el grupo de bajo riesgo, se recomienda iniciar con $15-25 \mathrm{kcal} / \mathrm{kg} / \mathrm{d}$ durante los primeros 3 días; al día 4 se debe incrementar a $30 \mathrm{kcal} /$ $\mathrm{kg} / \mathrm{d}$ y en los días 5-10 se debe alcanzar el máximo de los requerimientos necesarios. Para mantener un balance neutro de fluidos, se recomienda el aporte aproximado de 30-35 $\mathrm{ml} / \mathrm{kg} / \mathrm{d}$, sin necesidad de restringir el aporte de sodio $(15,16)$.

En el grupo de alto riesgo se recomienda iniciar el soporte nutricional a un máximo de $10-15 \mathrm{kcl} /$ $\mathrm{kg} / \mathrm{d}$, incrementar el aporte a $15-25 \mathrm{kcal} / \mathrm{kg} / \mathrm{d}$ para los días 4-5 y para el día 6 progresar a 30 $\mathrm{kcal} / \mathrm{kg} / \mathrm{d}$. A los 7-10 días, se debe alcanzar el aporte máximo de nutrientes. Con respecto al balance de fluidos, se recomienda aportar de los días 1-3 entre $25-30 \mathrm{ml} / \mathrm{kg} / \mathrm{d}$ y a partir del día 4 de $30-35 \mathrm{ml} / \mathrm{kg} / \mathrm{d}$. Además, se debe restringir el aporte de sodio $<1 \mathrm{mmol} / \mathrm{kg} / \mathrm{d}$ durante los primeros 7 días para evitar una sobrecarga de volumen $(15,16)$.

Finalmente, en el grupo de muy alto riesgo, se recomienda iniciar la alimentación con un aporte de $5-10 \mathrm{kcal} / \mathrm{kg} / \mathrm{d}$ durante los primeros 3 días, de los días 4 al 6 aumentar el aporte a $10-20 \mathrm{kcal} /$ $\mathrm{kg} / \mathrm{d}$; del día 7 al 9 progresar a $20-30 \mathrm{kcal} / \mathrm{kg} / \mathrm{d}$ y finalmente, aportar todos los requerimientos calóricos necesarios para el día 10. En cuanto al balance de fluidos, se recomienda aportar los primeros 3 días de $20-25 \mathrm{ml} / \mathrm{kg} / \mathrm{d}$, en los días 4-6 de $25-30 \mathrm{ml} / \mathrm{kg} / \mathrm{d}$ y a partir del día 7 de $25-35$ $\mathrm{ml} / \mathrm{kg} / \mathrm{d}$. Se debe restringir el sodio a $<1 \mathrm{mmol} /$ $\mathrm{kg} /$ día durante los primeros 10 días $(15,16)$.

Otras de las medidas que se recomiendan 
incluyen: administrar 200-300mg de tiamina vía oral antes de reiniciar la realimentación y por los primeros 5 días, con el objetivo de evitar alguna complicación neurológica; suplementar con multivitamínicos a todos los paciente por 10 días, reponer la deficiencia de los elementos traza que sean necesarios y no suplementar hierro durante los primeros 7 días en los grupos de riesgo, aun cuando estos presenten deficiencia $(15,16)$.

Adicionalmente, es necesario monitorizar los electrolitos séricos diariamente durante los primeros 3 días, debido a que se ha documentado que en esas primeras 72 horas el cuerpo pasa de un estado catabólico a uno anabólico, por lo que el riesgo de desarrollar SR es mayor. Posteriormente, se debe continuar con las mediciones cada 2 o 3 días $(15,16)$.

Finalmente, es importante recalcar la importancia de una examinación clínica diaria, con el fin de detectar signos o síntomas prematuros de sobrecarga de volumen o de falla orgánica, relacionados con el SR. Dicha examinación, debe incluir el monitoreo continuo del ritmo cardíaco de los pacientes en riesgo, especialmente en aquellas personas con historia de arritmias $(6,15,16)$.

\section{MANEJO DEL PACIENTE CON SÍNDROME DE REALIMENTACIÓN}

EI SR suele ser de rápida instauración, por lo que el primer paso para manejarlo correctamente se debe basar en su diagnóstico y en la capacidad de detectarlo de manera temprana. En términos generales, se dice que un paciente sufre de SR cuando durante las primeras 72 horas de haber iniciado el soporte nutricional, se detecta un descenso de fosfato $>30 \%$ de la línea de base o una concentración sérica $<0.6 \mathrm{mmol} / \mathrm{L}$; o si el valor de otros dos electrolitos diferentes se encuentra por debajo del rango normal $(15,16)$. A la hora de realizar este diagnóstico, es importante tener presente que el SR puede catalogarse como manifiesto 0 inminente. En el primer caso, se presentan alteraciones hidroelectrolíticas de la mano con síntomas clínicos; sin embargo, en el inminente caso, únicamente se observan las alteraciones hidroelectrolíticas (16).

Una vez que se detecta el SR en cualquiera de sus formas, se recomienda disminuir el aporte calórico diario de los pacientes e iniciar o intensificar (en caso de que ya se esté llevando a cabo) la reposición de electrolitos. Adicionalmente, si el paciente presenta algún signo o síntoma clínico concreto como por ejemplo, edemas o insuficiencia cardíaca, es necesario complementar el manejo de su padecimiento con el tratamiento específico de la manifestación asociada $(10,16)$.

Conclusiones

EI SR es una entidad que potencialmente puede causar mortalidad y secuelas graves, por lo que es de vital importancia reconocerlo ante el inicio de soporte nutricional en cualquier paciente desnutrido o con déficit en sus requerimientos diarios.

La identificación de pacientes de riesgo es fundamental para iniciar protocolos que eviten el soporte agresivo en periodos tempranos. Aunque en diferentes estudios no hay consenso entre el aporte energético, electrolítico y de macronutrientes que deben recibir los pacientes, la guía del NICE es la más aceptada y, por tanto, las recomendadas a utilizar en este escenario de rehabilitación nutricional, el cual, si se realiza de forma adecuada y precisa, previene la incidencia se SF, así como sus consecuencias una vez instaurado. 


\section{BIBLIOGRAFIA}

1. Temprano Ferreras JL, Bretón Lesmes I, de la Cuerda Compes C, Camblor Álvarez M, Zugasti Murillo A, García Peris P. Síndrome de realimentación. Revisión. Rev Clin Esp. 2005;205(2):79_ 86.

2. Mehanna HM, Moledina J, Travis J. Refeeding syndrome: What it is, and how to prevent and treat it. Vol. 336, BMJ. BMJ Publishing Group; 2008. p. 1495-8.

3. Araujo Castro M, Vázquez Martínez C. The refeeding syndrome. Importance of phosphorus. Med Clin (Barc). 2018;150(12):472-8.

4. Kraft MD, Btaiche IF, Sacks GS. Review of the refeeding syndrome. Nutr Clin Pract. 2005;20(6):625-33.

5. Crook MA, Hally V, Panteli J V. The importance of the refeeding syndrome. Vol. 17, Nutrition. Elsevier; 2001. p. 632-7.

6. NICE. NICE Clinical Guidline 32. Nutrition support for adults: oral nutrition support, enteral tube feeding and parenteral nutrition. 2017;(August 2017):1-37.

7. Rio A, Whelan K, Goff L. Occurrence of refeeding syndrome in adults started on artificial nutrition support: prospective cohort study. BMJ Open [Internet]. 2013 [cited 2020 Mar 3];3:2173. Available from: http://bmjopen.bmj.com

8. Crook MA. Refeeding syndrome: Problems with definition and management. Nutrition [Internet]. 2014;30(11-12):1448-55. Available from: http://dx.doi.org/10.1016/j.nut.2014.03.026

9. Stanga Z, Brunner A, Leuenberger M, Grimble RF, Shenkin A, Allison SP, et al. Nutrition in clinical practice - The refeeding syndrome: Illustrative cases and guidelines for prevention and treatment. Vol. 62, European Journal of Clinical Nutrition. Nature Publishing Group; 2008. p. 687-94.

10. Mehanna HM, Moledina J, Travis J. Refeeding syndrome: What it is, and how to prevent and treat it. Bmj. 2008;336(7659):1495-8.

11. Omar M, Nouh F, Younis M, Younis M, Nouri A, Ahmed D, et al. Volume-3; Issue-11. SAS J Med [Internet]. 2017 [cited 2020 Mar 3];307-12. Available from: http://sassociety.com/sasjm/

12. Boateng AA, Sriram K, Meguid MM, Crook M. Refeeding syndrome: Treatment considerations based on collective analysis of literature case reports. Nutrition [Internet]. 2010;26(2):156-67. Available from: http://dx.doi.org/10.1016/j.nut.2009.11.017

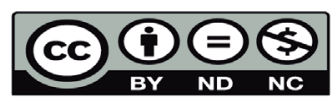


13. Tresley J, Sheean PM. Refeeding Syndrome: Recognition Is the Key to Prevention and Management. J Am Diet Assoc. 2008 Dec 1;108(12):2105-8.

14. Ahmed J, Khan LUR, Khan S, MacFie J. Refeeding syndrome: A literature review. Gastroenterol Res Pract. 2011;2011.

15. Rendón-Rodríguez R, Uresti-González II, Hernández-Ortega A, Torres-Wong AS. Síndrome de realimentación: estrategias para el abordaje nutricional. Nutr Clin Med. 2018;XII(2):95-108.

16. Friedli N, Stanga Z, Culkin A, Crook M, Laviano A, Sobotka L, et al. Management and prevention of refeeding syndrome in medical inpatients: An evidence-based and consensus-supported algorithm. Nutrition. 2018;47:13-20.

17 Boland K, Solanki D, Hanlon CO. Prevention and Treatment of Refeeding Syndrome in the Acute Care Setting. Irish Soc Clin Nutr Metab. 2013;(1):7-14. 